\title{
The Public Health System in India
}

\section{Radhika Kapur*}

Pedagogy and Organizational Culture in Nursery Schools, Delhi University, New Delhi, India

*Corresponding Author: Radhika Kapur, Pedagogy and Organizational Culture in Nursery Schools, Delhi University, New Delhi, India. Received: September 09, 2019; Published: October 14, 2019

DOI: $10.31080 /$ ASNH.2019.03.0504

\section{Abstract}

Health is considered to be a crucial factor for the social, economic, and overall development of the individual as well as the country. In this research paper, the public health system has been looked from the Indian perspective. The significance of health has been stated, what are the availability of healthcare resources, main areas that need to be taken under consideration within the healthcare system, what are the components of the public healthcare system, primary health care services, National Rural Health Mission and Health Insurance. All these factors have been highlighted in this research paper. When understanding these perspectives, the reader is able to know that there is a very close interconnection between health and the socio-economic developments; when an individual is healthy physically as well as mentally, only then he can get engaged in employment and accomplish something for the welfare of himself, his family and his country.

Keywords: Public Health; Healthcare; National Rural Health Mission; Health Insurance; Resources; Services

\section{Introduction}

Health is a positive state of well being in which harmonious development of physical and mental capacities lead to enjoyment of rich and complete life. Health is considered to be a vital aspect for the synchronized and integrated development of the individual, community and for the socio-economic development of the country. According to World Health Organization (WHO), health is the state of complete physical, mental and social well being and not merely the absence of the disease or illness [1].

Public health is the science and art of promoting health, preventing disease and prolonging life through the organized efforts of society (WHO). Public health is a social and physical concept that has an objective of improving the health conditions, prolonging life and quality of life among whole populations through the means of health promotion, disease prevention and other forms of health intervention. The Directive Principles of State Policy of Indian Constitution considers that the State shall regard increasing of the level of nutrition and standard of living amongst the people and improvement of public health as primary duties under Article 47. In addition, under Article 42, the State shall make provision for the availability of just and humanitarian conditions of work and of maternity relief. The health system in India is expected to perform with objectives based on these principles and evolve its spirit and structure in order to achieve these objectives [1].

\section{Availability of healthcare resources}

There have been considerable number of healthcare resources that have been available within the country and these resources have enhanced the public health system [2].

1. It has the largest number of medical colleges.

2. The medical colleges produce largest numbers of doctors in the developing world and these doctors are exported to many other countries of the world in order to render their contribution.

3. Looking at medicines; the country is the fourth largest producer of drugs in the world and is also the largest exporter of drugs in the world.

In India, despite the availability of these resources, experts and skilled practitioners in the medical field; majority of the citizens have limited access to the quality healthcare system, there are low levels of immunization, the wealthy individuals can afford high quality and expensive healthcare benefits but the poor people especially within the rural areas are unable to get even basic healthcare. The rates of hospitalization are six times higher amongst the rich as compared to the poor; the poor communities spend one-eighth of their income on healthcare. The government within the country spends much less amount on the healthcare, which is just one fifths, while the remaining portion is given by the ordinary citizens from their own pockets. This makes the healthcare system within the country to be one of the most privatized system in the world [2]. 
Main areas to be taken under consideration within the health care system

- Maternal Death and Disability: There has been an increase in the mortality rate amongst the mothers every year; these can be reduced if proper medical care and attention is provided to the mothers all the time when they are in need. This needs to be taken under consideration for the appropriate health care system in India [3].

- Infant and Child Mortality: An average IMR of 70 per 1000 live births and a CMR of 95 per 1000 live births are also very high as compared with 5-6 CMR in the developed nations. Most of these deaths are also preventable, if precautions are taken on time [3].

- HIV/AIDS: India has 5.1 million HIV/AIDS cases; this is causing major impediments in the path of improvements in the areas of health, economic and social development. Indicators of human development such as child mortality, literacy and food production are decreasing as the disease ravages families, communities, economies and health systems.

- Non-Communicable diseases: In India, the diseases that are non-communicable are on the rise such as there are 25 million cardiovascular diseases, 25 million cases of diabetes, 2.4 million cases of cancer and so on.

- Urban Health: Urbanization is considered to be an important demographic shift worldwide. Today, nearly $50 \%$ of the world population is urban, India has an urban population of 300 million and this presents $30 \%$ of the population. Urban health planning was implemented by the Government of India in the 9th five year plan. An urban slum growth rate of $5 \%$ is causing serious concern for the civic and health authorities in municipalities and towns. Health of urban poor and its implications on the entire urban population should be analyzed and the appropriate urban health policy should be formulated [3].

- Environmental Health: Contaminated water, pollution and poor health conditions are responsible for the spread of large number of diseases and health problems in India. There have been $9 \%$ of the deaths that have been caused due to poor hygiene and sanitation, an estimate of 27.4 million years of life is lost each year in India. Not much attention has been paid towards the improvement of these conditions; it is recommended that toilets should be established in rural areas, provisions must be made for clean drinking water, and cleanliness should be maintained within the surroundings in order to improve the environmental health.
- Healthcare Insurance: The contribution of the government towards the healthcare expenditure is only $20 \%$; therefore, out of pocket expenditure is $80 \%$. The cost of healthcare has been increasing rapidly and the large majority of the population cannot afford healthcare expenses. In India there are five forms of healthcare insurance- private insurance, social insurance, employer-provided cover, community insurance schemes and government healthcare spend. Only 3-4\% of our population is insured, insurance cover has grown by $100 \%$ in the last two years. It is estimated that about 160 million people will be covered by 2010 , which is less than $15 \%$ of the population [3].

- Support Services: Support services such as blood banking, ambulance services, communication, medical-social work, hospital waste management etc are very essential to provide good quality health and medical services. In the healthcare sector in India, these support services are lacking or they are not of good quality, in other words, there has been a high rate of incompetence in implementing the tasks. The problems arise mainly within the rural areas such as there is not sufficient availability of blood, ambulance service is unreliable, and medical infection control and waste disposal are in a pitiable condition. Thus there has to be formulation of appropriate steps and procedures for the enhancement of healthcare system [3].

\section{Components of public health care system}

The health care system consists of primary, secondary and tertiary care institutions that are operated by medical and paramedical personnel; medical colleges and paraprofessional training institutions to train the needed manpower and give the required academic input; program managers and authorities managing ongoing programs at central, state and district levels and health management information system consisting of a two way system of data collection, spread, analyses and response [4]. The public health system has to comprise of proper well established medical colleges that include skilled and trained practitioners, hospitals should be well equipped with trained and expert doctors and there should be availability of health benefits such as blood, medicines, medical equipment and so forth. Factors such as availability of clean drinking water, hygiene, sanitation, toilets are essential to maintain good health. There should be initiation of camps especially within the rural areas which may provide the facility of free medical check up for all the people of all age groups.

\section{Primary health care services}

The primary health care infrastructure provides the first level of contact between the population and health care providers. Com- 
prehending its significance in the delivery of health services, the centre, states and several government related agencies concurrently started creating primary health care infrastructure and manpower; this has resulted in significant amount of duplication of the infrastructure and manpower. The primary health care center consists of sub centers, primary health centers, community health centers, hospitals, rural family welfare centers, urban health posts, urban family welfare centers, district post partum centers, and sub district post partum centers funded by the department of family welfare, dispensaries and clinics, urban health services provided by municipalities, health care for central government employees provided by the Central Government Health Scheme (CGHS), hospitals and dispensaries of railways, defense and similar major departments make provision for healthcare services for their personnel, medical infrastructure, Employee's State Insurance Scheme (ESIS) hospitals and dispensaries providing health care to employees of industries, all hospitals even those providing secondary or tertiary care also provide primary health care services to rural and urban population and over three-fourths of the medical practitioners work in the private sector and majority of them cater to the primary health care requirements of the urban and rural population [4].

\section{National rural health mission}

The National Rural Health Mission (NRHM), launched in 2005, is the first health pro-gram in a "Mission Mode" to advance the health system and the health status of the people, especially for those who reside in the rural areas, and provide universal admittance to reasonable, affordable and quality healthcare which is responsible and at the same time approachable to the requirements of the people. The program is an all-inclusive package of pro-motive, preventive, curative and rehabilitative services to be delivered to the community through a process of intersectoral co-ordination with other service departments and active community participation. Various national programs like immunization, tuberculosis control, leprosy elimination, cancer control etc. have been integrated under the NRHM program that also addresses the social determinants of health and delivery of the same with the active participation of Panchayat Raj Institutions (local governance) for its sustainability. The program will help achieve goals set under the National Health Policy and the Millennium Development Goals. It also seeks to regenerate and incorporate local health traditions of medicine (Ayurveda, Yoga and Naturopathy, Unani, Siddha and Homoeopathy: AYUSH) into the public health system. Health is for the first time being seen as a constituent of development package [5].

The NRHM sought to increase public spending on health, lessen regional imbalances in health infrastructure, collect resources, integrate various organizational structures and vertical national programs, decentralize and achieve district management of health programs, and turn community health centers into functional hospitals meeting certain standards. The NRHM has an exceptional focal point on rural areas in 18 States which have weak public health indicators and/or weak infrastructure [5].
At the village level the government has promoted the concept of having an accredited female social health activist (ASHA) in order to make possible the household right to use healthcare. Village Health Committees of the Panchayat Raj are responsible for putting in place Village Health Plans. The NRHM also calls for the preparation and implementation of an inter-sectoral District Health Plan organized by the District Health Mission. Such a plan should include provisions for drinking water, sanitation, hygiene and nutrition. The NRHM also has provisions for capacity building aimed at intensification of the National, State and District Health Missions, for example through data collection, assessment and review for evidence-based planning, monitoring and supervision [5].

\section{Health insurance}

Health Insurance in India is at its infant stage. There are several insurance schemes operated by the Central and State governments, such as the Rashtriya Swasthya Bima Yojana (RSBY) which targets Below Poverty Line (BPL) families, the Employees' State Insurance Scheme (ESIS) and the Central Government Health Scheme (CGHS). There are also public and private insurance companies as well as several community-based organizations. An estimated 300 million people are believed to be covered by health insurance in India. Of these, approximately 243 million are covered by different forms of government-sponsored insurance schemes while approximately 55 million rely on commercial insurers [5].

The RSBY offers an example in which the State governments, through a bidding process, select a public or private insurance company to provide health insurance for the target group. Under the scheme, the beneficiaries also have the freedom to choose between participating public or private hospitals when deciding where to receive healthcare. Experience from the functioning of the RSBY shows that insurance companies, especially in the private sector, have been successful at controlling costs, for example, control of fraudulent means, man-aging customer complaints, and tracking the costs and the quality of the services provided by participating hospitals. However, despite this fact, in the ongoing discussion about how to organize the Government of India's planned Universal Health Coverage scheme, it has been suggested that the purchases of all healthcare services be managed either by the Central or State governments through the respective Department of Health or by other government agencies, and not by insurance companies or other independent agencies [5].

\section{Conclusion}

Health is a crucial factor and is relevant amongst the community of all kinds of individuals whether they are residing in urban or rural areas, they are of different age groups, different genders and so forth. In India, programs and procedures have been formulated to develop the public healthcare system such as NRHM, which has the main objective of improving the health status of the individuals particularly in rural areas; but in spite of all these benefits the public healthcare system is still in a backward condition. The public healthcare system is more undeveloped within the rural areas as 
compared to the urban; the illnesses and the spread of diseases are more prevalent due to the factors such as contaminated water, lack of sanitation, hygiene, different kinds of pollution, air, water, absence of proper civic amenities and villagers also due to lack of awareness get more prone to usage of tobacco and alcohol.

There have been establishment of proper medical institutions, hospitals, dispensaries, clinics and free medical camps with skilled and trained medical practitioners so that they can contribute effectively towards the development of the public healthcare system within the country. The major problem that the individuals are facing is that the government is contributing only $20 \%$ towards the medical and health benefits, the rest of the major portion the individuals have to pay out of their own pocket; in order to solve this problem, unaffordable and inaccessible, there have been establishment of medical and healthcare centers in all areas so that people have access to them easily and health insurance schemes have been established so that people get some benefits regarding their healthcare and medical treatment. Finally, it can be stated that in order to effectively maintain the healthcare services within the country, major areas that should be taken under consideration are maternal death and disability, infant and child mortality, HIV/ AIDS, non-communicable diseases, urban health, environmental health, healthcare insurance and support services.

\section{Bibliography}

1. Public Health System in India: An Introduction and Evolution (n.d.).

2. Health System in India: Crises and Alternatives. National Coordination Committee. Jan Swasthya Abhiyan (2006).

3. Ramani KV and Dileep M. "Health System in India: Opportunities and Challenges for Improvements". Indian Institute of Management, Ahmedabad, India (2005).

4. Public Health Care System (2000).

5. India's Healthcare System - Overview and Quality Improvements (2013).

Volume 3 Issue 11 November 2019

(C) All rights are reserved by Radhika Kapur. 